\title{
Bulk Composite Nanomaterial with Multiwall Carbon Nanotubes
}

\author{
Levan Ichkitidze $^{1 *}$, Vitally Podgaetsky $^{1}$, Alexander Prihodko ${ }^{1}$, Sergei Selishchev $^{1}$, Eugenie Blagov $^{2}$, \\ Vyacheslav Galperin $^{3}$, Yuri Shaman ${ }^{3}$, Lyudmila Tabulina ${ }^{4}$ \\ ${ }^{1}$ National Research University of Electronic Technology (MIET), Moscow, Russia; ${ }^{2}$ Institute of Nanotechnology of Microelectronics \\ of the Russian Academy of Sciences (INME RAS), Moscow, Russia; ${ }^{3}$ Scientific Manufacturing Complex "Technological Centre", \\ Moscow, Russia; ${ }^{4}$ Belarusian State University of Informatics and Radioelectronics, Minsk, Belarus. \\ Email: *leo852@inbox.ru
}

Received July $17^{\text {th }}, 2012$; revised August $16^{\text {th }}, 2012$; accepted September $17^{\text {th }}, 2012$

\begin{abstract}
The properties of the composite nanomaterials (CNM) based on bovine serum albumin (BSA) and multi-walled carbon nanotubes (MWCNT), both functionalized and non-functionalized, were investigated. In order to obtain the solid-state bulk CNM from the ultradispersed aqueous solutions of $25 \mathrm{wt} \%$ BSA and $(0.0015-0.04) \mathrm{wt} \%$ MWCNT, the methods of nanotechnology and laser technology were used. It is revealed that the CNM density is $10 \%-20 \%$ higher than that of water and the hardness is higher than that of BSA by a factor of 3 - 6 times. An increase in hardness $H_{\mathrm{v}}$ (by Vickers) of CNM correlated with an increase in the concentration of MWCNT, and $H_{\mathrm{v}}$ reached $\sim 300 \mathrm{MPa}$ for the case of the non-functionalized MWCNT, while for the case of the functionalized MWCNT, i.e. $\mathrm{MWCNT}_{\mathrm{f}}, H_{\mathrm{v}}$ was $25 \%$ lower.
\end{abstract}

Keywords: Carbon Nanotubes; Functionalization; Albumine; Biocompatible Composite Nanomaterial

\section{Introduction}

High mechanical properties are observed for carbon nanotubes (CNT), and they are recordly high for singlewall CNT (SWCNT), for example, the Young modulus is of about $5 \mathrm{TPa}$, which is much higher than that of high-strength steels. Hence, the MWCNT addition to the material in a very low concentration $(\sim 0.1 \%)$ significantly improves the mechanical properties for most materials including polymers and ceramics, which is one of the reasons for the rise of interest in the wide application of CNT in modern technology and medicine [1].

The examined studies, regarding the risk and safety of CNT, as well as nanomaterials and products based on them, imply several important inferences [2-7]: CNT toxicity depends on their treatment of various contaminants, including the catalytic metals; the toxicity of SWCNT is higher than that of MWCNT; the toxic manifestation of SWCNT is less than that of the same dose of asbestos particles, placed under the skin of experimental animals (mice); the functionalized CNT are much less toxic than non-functionalized. According to the latter conclusion, it is obvious that preference should be given to the functionalized CNT when creating nanomaterials based on CNT for biomedical applications. Partly this approach was used in [8-11] for obtaining

"Corresponding author. bulk composite materials BSA + MWCNT and studying their mechanical properties since albumin functionalizes CNT [12]. On the other hand, bulk composite nanomaterials BSA + MWCNT are of independent interest, when the used MWCNT are preexposed to functionalization. The purpose of this study was to investigate the mechanical properties of these materials.

\section{Samples Preparation and Measurment Techniques}

The studied CNM consisted of the two main components: albumin, and carbon nanotubes. Albumin constitutes of about $60 \%$ of blood plasma proteins of humans and animals, and it has been well studied at the atomic and structural level. As well as BSA, human serum albumin (HSA) is widely used in medical practice, but HSA is less stable than BSA. The choice of BSA was determined by its high biocompatibility, wide accessibility, and stability of its characteristic parameters.

MWCNT were preferred CNT since they are less toxic and more accessible as opposed to SWCNT.

The bimetallic catalyst $\mathrm{Fe}-\mathrm{Mo} / \mathrm{MgO}$ was used to obtain MWCNT. MWCNT were synthesized at $900^{\circ} \mathrm{C}$ for 40 minutes in the $\mathrm{Ar} / \mathrm{CH} 4$ flow. After the synthesis, the material was subjected to multistage chemical processing resulted in MWCNT covered with functional groups that 
contribute to the formation of a stable aqueous suspension of MWCNTf. The MWCNT fraction in the final material after the treatment was $95 \mathrm{wt} \% \pm 1 \mathrm{wt} \%$.

Stages of functionalization of MWCNT are described in detail in $[13,14]$, but we here note some main procedures allowing us to obtain stable water MWCNT suspensions:

- Annealing of the MWCNT-containing material in air for $1.5 \mathrm{~h}$ at $350^{\circ} \mathrm{C}$;

- Room-temperature treatment in hydrochloric acid for $24 \mathrm{~h}$ to remove catalyst particles;

- Treatment in concentrated nitric acid at $140^{\circ} \mathrm{C}$ for $1 \mathrm{~h}$;

- MWCNT treated with mineral acids were washed with water to $\mathrm{pH}=5$ by decantation and dried on a water bath and then in a drying oven at $120^{\circ} \mathrm{C}$ to a constant weight;

During performing the acidic treatment, carboxyl $(\mathrm{O}=\mathrm{C}-\mathrm{OH})$, carbonyl $(\mathrm{C}=\mathrm{O})$, and aldehyde groups

$(\mathrm{O}=\mathrm{C}-\mathrm{H})$, which prevent the coagulation of MWCNT in the solution and increase the stability of $\mathrm{MWCNT}_{\mathrm{f}}$ in water, are formed.

In this work, we used the methods of obtaining the CNT, which are described in [8-11], where the combined nano- and laser technologies were used. At that, numerous technological parameter as stirring duration, power and duration of the ultrasonic treatment, values of $C$, power density and duration of the laser treatment, temperature and time of the additional drying of fabricated CNT, etc. were used.

To form the CNT, we performed the preliminary procedures:

1) The BSA powder was dissolved in distilled water. The obtained solution was dispersed in a magnetic stirrer for $1-2 \mathrm{~h}$ and in the ultrasonic bath at $\leq 40^{\circ} \mathrm{C}$, decanted, and filtered.

2) Powders of MWCNT or carbon black K-354 with the concentration of $0.0015 \mathrm{wt} \%-0.04 \mathrm{wt} \%$ were added into the aqueous BSA solution, while ash was added to the reference samples in the same proportion.

3) Obtained suspensions of $25 \mathrm{wt} \% \mathrm{BSA}+\mathrm{MWCNT}$, $25 \mathrm{wt} \% \mathrm{BSA}+$ carbon black K-354, and $25 \mathrm{wt} \% \mathrm{BSA}+$ $\mathrm{MWCNT}_{\mathrm{f}}$ were treated similarly to point 1 .

In this study, we used the methods for obtaining bulk CNM, which are described in [8-11], where nanotechnology and laser technology are used in combination. During preparation of the samples, numerous technological parameters were varied including the power density, the duration time of laser irradiation, parameters of preparation of aqueous solutions BSA + MWCNT (the duration time of mixing, the power level and duration time of ultrasonic treatment, etc.), the MWCNT concentration level, temperature, and the extra drying duration time for the prepared CNM.

The prepared suspensions were irradiated using a di- ode laser (the wavelength of the radiation $970 \mathrm{~nm}$, the radiation power changes in the interval $0-20 \mathrm{~W}$ ) until the water evaporation and the CNM formation with varying the consistency from rubber-like to a solid state. Later they were dried in air at $30^{\circ} \mathrm{C}-35^{\circ} \mathrm{C}$. The indication of sufficiently high quality of the final product was the absence of whitish areas of denatured albumin in it.

Bulk samples obtained with the use of laser irradiation behave differently during storage in normal conditions: CNM with MWCNT or $\mathrm{MWCNT}_{\mathrm{f}}$ retain their form and strength for a year or longer, while BSA or BSA+carbon black K-354 decompose into individual flakes of material in a few hours $(\leq 16 \mathrm{~h})$, similarly to the process occurring during normal drying of their aqueous solutions and suspensions.

The relative optical density (absorption coefficient) with respect to distilled water and air was measured for all the suspension samples with the use of a photometer. The solid-state CNM samples of were $\sim 1 \mathrm{~g}$ in weight, while the CNM density $\rho$ was measured by the method of hydrostatic weighing. $H_{\mathrm{v}}$ was determined by the Vickers scale using a microhardnessmeter.

\section{Experimental Results and Discussion}

On the Figure 1 presents the typical SEM images for the studied CNM.

They testify to the following: CNM with MWCNT are inhomogeneous as in some places formed clusters of the MWCNT (Figure 1(a)), while in other places MWCNT are virtually absent. CNM to $\mathrm{MWCNT}_{\mathrm{f}}$ are homogeneousfunctionalized carbon nanotubes are observed everywhere, and they do not form clusters (Figure 1(b)). SEM picture of the last case is similar to the picture, when carbon nanotubes are homogeneously distributed in the CNM [15].

Studies were performed for three series of samples consisting of the following parts: I-BSA and BSA + carbon black K-354; II-BSA-based CNM and the nonfunctionalized MWCNT, i.e. BSA + MWCNT; IIIBSA-based CNM and the functionalized MWCNT, i.e. $\mathrm{BSA}+\mathrm{MWCNT}_{\mathrm{f}}$. In all series of the samples, the concentrations of components were as follows: BSA $25 \mathrm{wt} \%$ and carbon additions $0.0015 \mathrm{wt} \%-0.04 \mathrm{wt} \%$.

Figure 2 shows the CNM surfaces examined using an optical microscope. In the first case, i.e. for the CNM sample with a low concentration level $C \approx 0.0030 \mathrm{wt} \%$ of non-functionalized MWCNT (Figure 2(a)), the clusters isolated from the main matrix are visible. Their average size is of about $\sim 20 \mu \mathrm{m}$ and, apparently, they are formed as a result of agglomeration of the nanotubes. In another case, for $\mathrm{CNM}_{\text {with }} \mathrm{MWCNT}_{\mathrm{f}}$ (Figure 2(b)), the matrixisolated clusters are hardly visible since their size is much smaller than that in the first case. Figure 2(c) shows the pattern of the sample surface viewed using a 


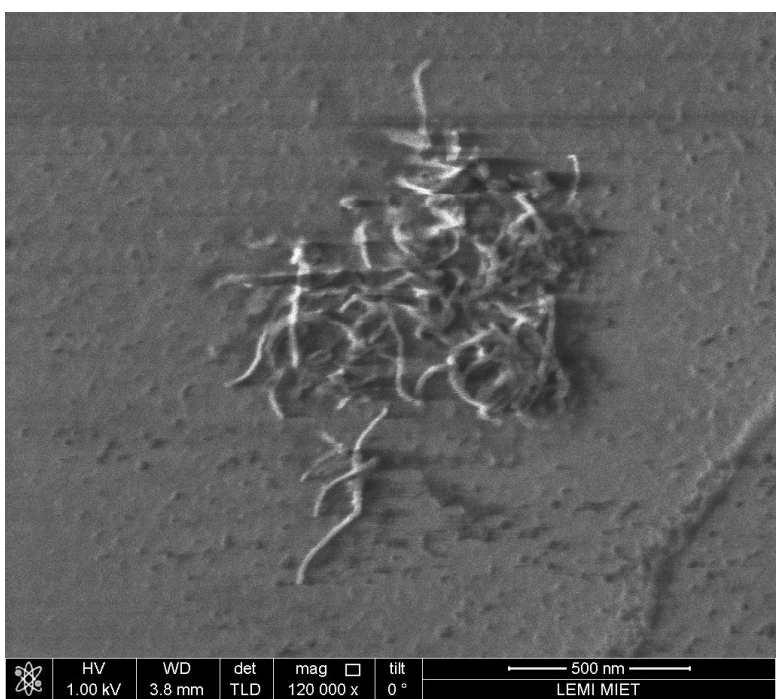

(a)

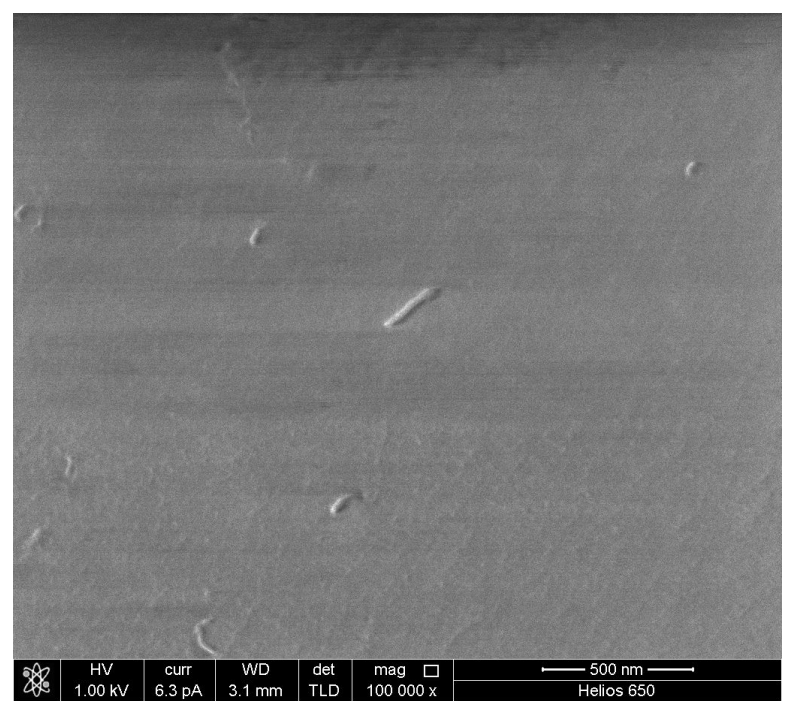

(b)

Figure 1. Typical SEM images for CNMs with non-functionalized ((a) MWCNT) and functionalized; ((b) MWCNT $_{f}$ carbon nanotubes. The pictures are the same scale $500 \mathrm{~nm}$.

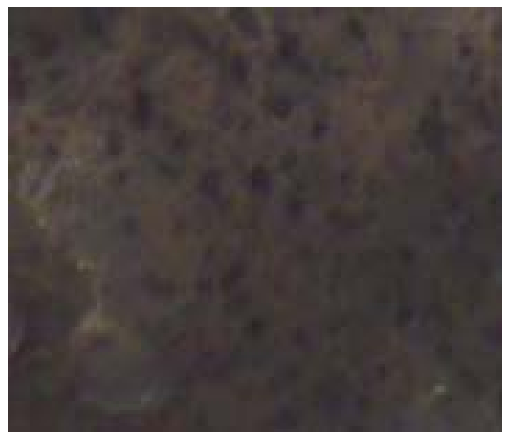

(a)

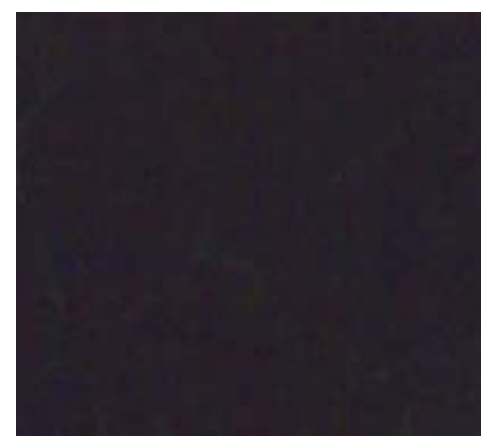

(b)

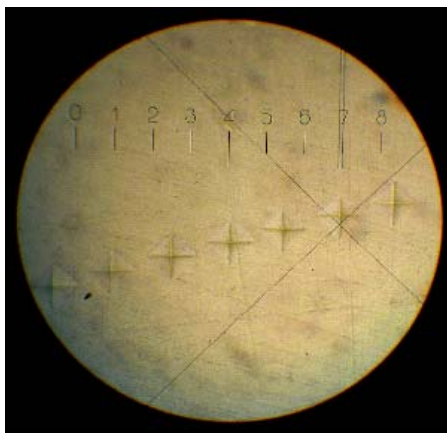

(c)

Figure 2. The view of the surface of several samples: (a) CNM, 25\% BSA $+0.0030 \mathrm{wt} \%$ MWCNT (optical microscope, magnification $\times 150$ ); (b) CNM, 25\% BSA + 0.0030 wt $\%$ MWCNT $_{f}($ optical microscope, magnification $\times 150)$; (c) The view of a typical surface in a microhardnessmeter microscope, scale division of $30 \mu \mathrm{m})$.

microhardnessmeter. It is evident that the sizes of the marks of the diamond indenter do not vary significantly, which also implies the homogeneity of the samples.

The results of optical measurements (range wavelengths 380 - $990 \mathrm{~nm}$ ) for the aqueous suspensions BSA + MWCNT, BSA + MWCNT and the surfaces of the bulk CNM based thereon correlate to one another in samples with MWCNT observed low absorption coefficients (translucent) and large cluster formation, separated from the main matrix; in the samples with $\mathrm{MWCNT}_{\mathrm{f}}$ realized high absorption coefficients (opaque) and the absence of clusters. Apparently, this ratio emphasizes the fact that the latter samples are more homogeneous than the former ones.

The Table 1 shows the measured mechanical properties of several samples. Ratios between the properties are typical for all the CNM samples studied.

For example, the total mass loss due to the moisture (water) loss while cooking and drying the samples, a higher density $(\sim 20 \%)$ of the final product relative to BSA and water, and a high ratio of the values of $H_{\mathrm{v}}$ for $\mathrm{CNM}$ and $\mathrm{BSA}-4$ - 6. It should be emphasized that selective measurements of the tensile strength of the CNM samples showed the values 7 - 10 times smaller than the respective values of $H_{\mathrm{v}}$ which is typical for ceramic materials.

Thus, minor MWCNT additions to the biological albumin material may facilitate to obtain the bulk composite biocompatible nanomaterial with high mechanical properties close to the natural porous bone. A higher value $(30 \%)$ of hardness of the nanomaterial based on BSA + MWCNT relative to the nanomaterial BSA $+\mathrm{MWCNT}_{\mathrm{f}}$ is, apparently, neutralized with a higher degree of safety of the latter ones. We can expect that further variation of numerous technological parameters of preparation of bulk nanomaterials BSA + $\mathrm{MWCNT}_{\mathrm{f}}$ improve their me- 
Table 1. Parameters of the samples consisting of $25 \mathrm{wt} \%$ BSA and additions of carbon particles at concentration of 0.035 wt\%.

\begin{tabular}{lcccc}
\hline \multirow{2}{*}{ Samples/options } & \multicolumn{2}{c}{ Series I } & Series II & Series III \\
\cline { 2 - 5 } & BSA & BSA + carbon black & BSA + MWCNT & BSA + $^{*}$ MWCNT $_{\mathrm{f}}$ \\
\hline The ratio of the mass of NCM before and after drying & 3.8 & 3.9 & 3.6 & 3.5 \\
Density, $\rho\left(\mathrm{g} / \mathrm{cm}^{3}\right)$ & 1.03 & 1.04 & 1.25 & 1.24 \\
Hardness, $H_{\mathrm{v}}(\mathrm{MPa})$ & 50 & 50 & 300 & 230 \\
\hline
\end{tabular}

chanical parameters.

\section{Conclusions}

The studies of the properties of the bulk biocompatible composite nanomaterials show that: the density of the nanocomposites is $10 \%-20 \%$ higher than the density of the constituent materials - albumin and water; the hardness $(200-300 \mathrm{MPa})$ of the nanocomposites is $4-6$ times higher than that of albumin and is close to the hardness of the bone issue ( 500 MPa).

It is established that the bulk nanomaterials with functionalized multi-walled carbon nanotubes are more homogeneous (no cluster separated from the matrix). They have higher absorption coefficients (opaque) and $\sim 25 \%$ lower hardness than the nanomaterials with non-functionalized multiwall carbon nanotubes (present cluster separated from the matrix, translucent).

The studied biocompatible nanomaterials with improved mechanical properties may be potentially useful as functional materials for biomedicine (e.g., surgical implants), as well as very lightweight materials for aerospace structures.

\section{Acknowledgements}

We thank collaborators of the Laboratory of Strength and Dynamic Tests, the Center of Collective Use "Nanotechnologies in Electronics" of National Research University of Electronic Technology (MIET) and Researchand-production firm "DELTARUS" for support in the work and useful advices.

This work is partially funded by Ministry of Education of the Russian Federation: state contract No. 16.426.11. 0043, September 12, 2011 and by the Foundation for Promotion of Small Enterprises in Science and Technology (contract 9610p/16276).

\section{REFERENCES}

[1] N. Grobert, "Carbon Nanotubes-Becoming Clean," Materials Today, Vol. 10, No. 1-2, 2007, pp. 28-35. doi:10.1016/S1369-7021(06)71789-8

[2] L. P. Ichkitidze and I. V. Komlev, "Carbon Nanotubes and Composite Nanomaterials: Toxicity," Lasers in Sci- ence, Engineering, Medicine, No. 21, 2010, pp. 103-113.

[3] J. Kolosnjaj-Tabi, K. B. Hartman, S. Boudjemaa, J. S. Ananta, G. Morgent, H. Swarc, L. J. Wilson and F. Moussa, "In Vivo Behavior of Large Doses of Ultrashort and Full-Length Single-Walled Carbon Nanotubes after Oral and Intraperitoneal Administration to Swiss Mice," ACS Nano, Vol. 4, No. 3, 2010, pp. 1481-1492. doi:10.1021/nn901573w

[4] K. Donaldson, R. Aitken, , L. Tran, V. Stone, R. Duffin, G. Forrest and A. Alexander, "Carbon Nanotubes: Review of Their Properties in Relation to Pulmonary Toxicology and Workplace Safety," Toxicological Science, Vol. 92, No. 1, 2006, pp. 5-22. doi:10.1093/toxsci/kfj130

[5] S. Magrez, V. Kasas, V. Salicio, N. Pasquier, J. W. Seo, M. Celio, S. Catsicas, B. Schwaller and L. Forro, "Cellular Toxicity of Carbon-Based Nanomaterials," Nano Letters, Vol. 6, No. 6, 2006, pp. 1121-1125.

doi:10.1021/n1060162e

[6] S. Koyama, Y.-A. Kim, T. Hayashi, K. Takeuchi, C. Fujii, N. Kuroiwa, H. Koyama, R. Tsukahara and M. Endo, "In Vivo Immunological Toxicity in Mice of Carbon Nanotubes with Impurities," Carbon, Vol. 47, No. 5, pp. 13651372. doi:10.1016/j.carbon.2009.01.028

[7] J. Muller, F. Huaux, N. Moreau, P. Misson, J. F. Heilier, M. Delos, M. Arras, A. Fonseca, J. B. Naqy and D. Lison, "Respiratory Toxicity of Multi-Walled Carbon Nanotubes," Toxicology and Applied Pharmacology, Vol. 207, No. 3, 2005, pp. 221-231. doi:10.1016/j.taap.2005.01.008

[8] I. A. Andreeva, V. B. Bagratashvili, L. P. Ichkitidze, V. M. Podgaetsky, O. V. Ponomareva, V. S. Savranskii and S. V. Selishchev, "Determination of Mechanical Properties of Biocompatible Three-Dimensional Nanocomposites Manufactured Using Laser Methods," Biomedical Engineering, Vol. 43, No. 6, 2009, pp. 241-248. doi:10.1007/s10527-010-9134-4

[9] L. P. Ichkitidze, V. M. Podgaetsky, O. V. Ponomareva and S. V. Selishchev, "Mechanical Properties of Bulk Nanocomposite Obtained by Laser Irradiation," Izvestiya VUZov. Physica (Russia), Vol. 53, No. 3/2, 2010, pp. 125-129.

[10] S. A. Ageeva, V. I. Eliseenko, A. Yu. Gerasimenko, L. P. Ichkitidze, V. M. Podgaetsky and S. V. Selishchev, "Possible Medical Application of Laser Nanoengineering," Biomedical Engineering, Vol. 44, No. 6, 2011, pp. 233236. doi:10.1007/s10527-011-9195-z

[11] A. Yu. Gerasimenko, O. V. Gubar'kov, L. P. Ichkitidze, V. M. Podgaetskii, S. V. Selishchev and O. V. Ponomareva, "Nanocomposite Solder for Laser Welding of Biol- 
ogical Tissues," Semiconductors, Vol. 45, No. 13, 2011, pp. 1713-1718. doi:10.1134/S1063782611130112

[12] X. Zhao, R. Liu, Z. Chi, Y. Teng and P. Qin, "New Insightsinto Behavior of Bovine Serum Albumin Adsorbed onto Carbon Nanotubes: Comprehensive Spectroscopic Studies," Journal of Physical Chemistry B, Vol. 114, No. 16, 2010, pp. 5625-5631.

[13] Y.-P. Sun, K. Fu, Y. Lin and W. Huang, "Functionalized Carbon Nanotubes: Properties and Applications," Ac- counts of Chemical Research, Vol. 35, No. 12, 2002, pp. 1096-1104. doi:10.1021/ar010160v

[14] N. V. Glebov and A. A. Nechitailov, "Functionalization of the Surfacemultiwalled Carbon Nanotubes," Technical Physics Letters (Russia), Vol. 36, No. 19, 2010, pp. 8-15.

[15] M. Berkei, "Conductive Coatings Using Carbon Nanotubes: A Fascinating Material for the Coating Producer's Toolbox," CHEManager Europe, 2011, p. 10. www.chemanager-online.com/en/tags/coatings 